\title{
Adaptive threshold to compensate the effect of muscle fatigue on elbow-joint angle estimation based on electromyography
}

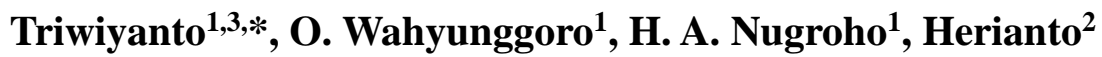 \\ ${ }^{1}$ Department of Electrical Engineering \& Information Technology, \\ Universitas Gadjah Mada, Yogyakarta, Indonesia \\ ${ }^{2}$ Department of Mechanical \& Industrial Engineering \\ Universitas Gadjah Mada, Yogyakarta, Indonesia \\ ${ }^{3}$ Department of Electromedical Engineering, \\ Politeknik Kesehatan Surabaya, Kementerian Kesehatan, Indonesia \\ Phone: +628155126883; Fax: +62315025609 \\ *Email: triwiyanto123@gmail.com
}

\begin{abstract}
Muscle fatigue is a major problem in the study based on electromyography (EMG) signal. When the muscle in the fatigue condition, the power of the EMG signal increases significantly. This phenomenon can be a problem in the elbow-joint angle estimation. The purpose of this study is to develop a method in order to compensate the effect of the muscle fatigue on the elbow-joint angle estimation based on time domain features using EMG signal. The EMG signal was collected from biceps while the subjects performed a fatiguing motion of flexion and extension. The EMG was extracted using four time-domain features, namely zero crossing (ZC), sign slope change (SSC), Wilson amplitude (WAMP) and myopulse percentage rate (MYOP). The yielded features were filtered using second order Butterworth low pass filter. In the proposed method, to compensate the effect of the muscle fatigue, the RMS of the EMG signal was calculated for every cycle and used it as a threshold value of the features. The results show that the proposed method is able to compensate the effect of muscle fatigue with a consistent root mean square error (RMSE). The improvement of the performance ranges between $17.41 \%$ and $37.9 \%$ (for all adaptive features).
\end{abstract}

Keywords: Electromyography; feature extraction; elbow-joint angle estimation; muscle fatigue; low pass filter.

\section{INTRODUCTION}

Recently, electromyography (EMG) $[1,2]$ is used a lot in the human-machine interaction such as virtual reality, prosthetic devices, exoskeleton and teleoperation [3-6]. Joint-angle estimation is essential in the development of the human-machine interaction to estimate the position of the limb or to learn the human motion $[7,8]$. The following are the examples of the estimation of an elbowjoint angle using a machine learning method. Tang used an artificial neural network (ANN) [9] with the back-propagation algorithm to estimate the elbow-joint angle from the EMG features [10]. Lee proposed a method to estimate knee joint angle using least square support vector machine (LS-SVM) [11]. Previous researchers were also used Hill-based muscle model to estimate the elbow-joint angle [13]. Pau used Genetic Algorithm (GA) to optimize the estimate, however, the GA is a high time consuming to perform an optimization [12]. Kiguchi used a neuro-fuzzy algorithm to estimate the force from EMG to control an upper limb exoskeleton [14]. However, the problem in elbow joint 
angle estimation which involves machine learning based methods is that the method requires a learning stage to recognize the EMG pattern. Previous studies also developed a non-machine learning-based method to estimate the elbow-joint angle and force using EMG signal [15-18]. However, the problem and limitation are that the previous studies have not considered the effect of the muscle fatigue to the performance of the estimation.

When a human performs a repetitive and intense motion, the muscle fatigue might occur. This muscle fatigue can be categorized as physiological fatigue or localized muscle fatigue which refers to the failure of the muscle to sustain the force [19]. Muscle fatigue could be detected accurately based on invasive methods such as blood test and examination of the body tissue. However, those methods are not in accordance with the experiment which has to be observed continuously. Previous studies were preferred to use a non-invasive method for detecting the muscle fatigue using EMG signal $[19,20]$. Since the muscle fatigue caused the frequency and amplitude of the EMG signal to change [21-23], a new method has to be introduced to compensate the effect of muscle fatigue so that the estimation can be maintained. Na proposed a method to estimate the elbow joint force using muscle twitch model [24]. He found that the method can compensate the effect of muscle fatigue in the force estimation. Thilina developed a method to compensate the effect of muscle fatigue on the elbow joint angle estimation which is used to control upper limb exoskeleton [25]. In the study, he used the mean power frequency (MPF) to detect muscle fatigue and to adjust the weight of the neuro-fuzzy. The MPF was obtained after the EMG signal was transformed into a frequency domain using fast Fourier transform (FFT). Therefore, the feature such as MPF which based on frequency domain needs a high computation time to process.

In addition to the features based on the frequency domain, the time domain features are the most popular used to extract the EMG signal into some information, related to the elbow-joint angle $[3,10,26]$. Time domain features are divided into three categories, namely based on energy, complexities, and frequency [27]. In the time domain features based on frequency, we can get the frequency content of the EMG signal without any transformation such as FFT. These features are sign slope change (SSC), zero crossing (ZC), Wilson amplitude (WAMP) and myopulse percentage rate (MYOP). The result of these features is determined by the amplitude of the EMG signal and a threshold parameter. Due to the effect of muscle fatigue, the amplitude of EMG signal increases significantly [24]. In this case, the changes of the EMG signal can be detected by using root mean square (RMS) feature and further, it is used to modify the threshold parameter of the SSC, ZC, WAMP, and MYOP features.

According to the problems, the purpose of the study is to develop a method to compensate the effect of the muscle fatigue on the elbow-joint angle estimation based on EMG signal. The proposed method consists of time domain features extraction, low pass filter, and an adaptive threshold. The specific objectives of this study are: (1) to evaluate the performance of the elbowjoint angle estimation for both the standard method and the proposed method using standard parameter of root mean square error (RMSE), (2) to perform a statistical analysis in order to find the significant difference of performance between the proposed and the standard method using pair samples T-test.

\section{METHODS AND MATERIALS}

\section{Data Collecting}

The experiment protocol of this study had been approved by Ethics Committee of Health Polytechnic of Surabaya, Ministry of Health, Indonesia (No: 035/S/KEPK/V/2017). Ten healthy male subjects were involved in this study. First of all, the subjects read and filled the form of consents to understand the experimental protocols. Generally, in the flexion and extension motion, the muscle groups affected the EMG signal are biceps and triceps [29]. The previous study has revealed that biceps are more active to generate EMG signal than that from triceps [30]. Therefore, 
in this study, the EMG signal was only recorded from biceps muscles using a pair of disposable electrode (Ag/AgCl, size: 57 x $48 \mathrm{~mm}$, Ambu, Blue Sensor R, Malaysia) and one common electrode as the common ground. The placement of the electrodes was in accordance to SENIAM [31]. In order to assess the muscle fatigue, the subjects were in the standing position and held an exoskeleton frame (Figure 1) with a two-kg of load.

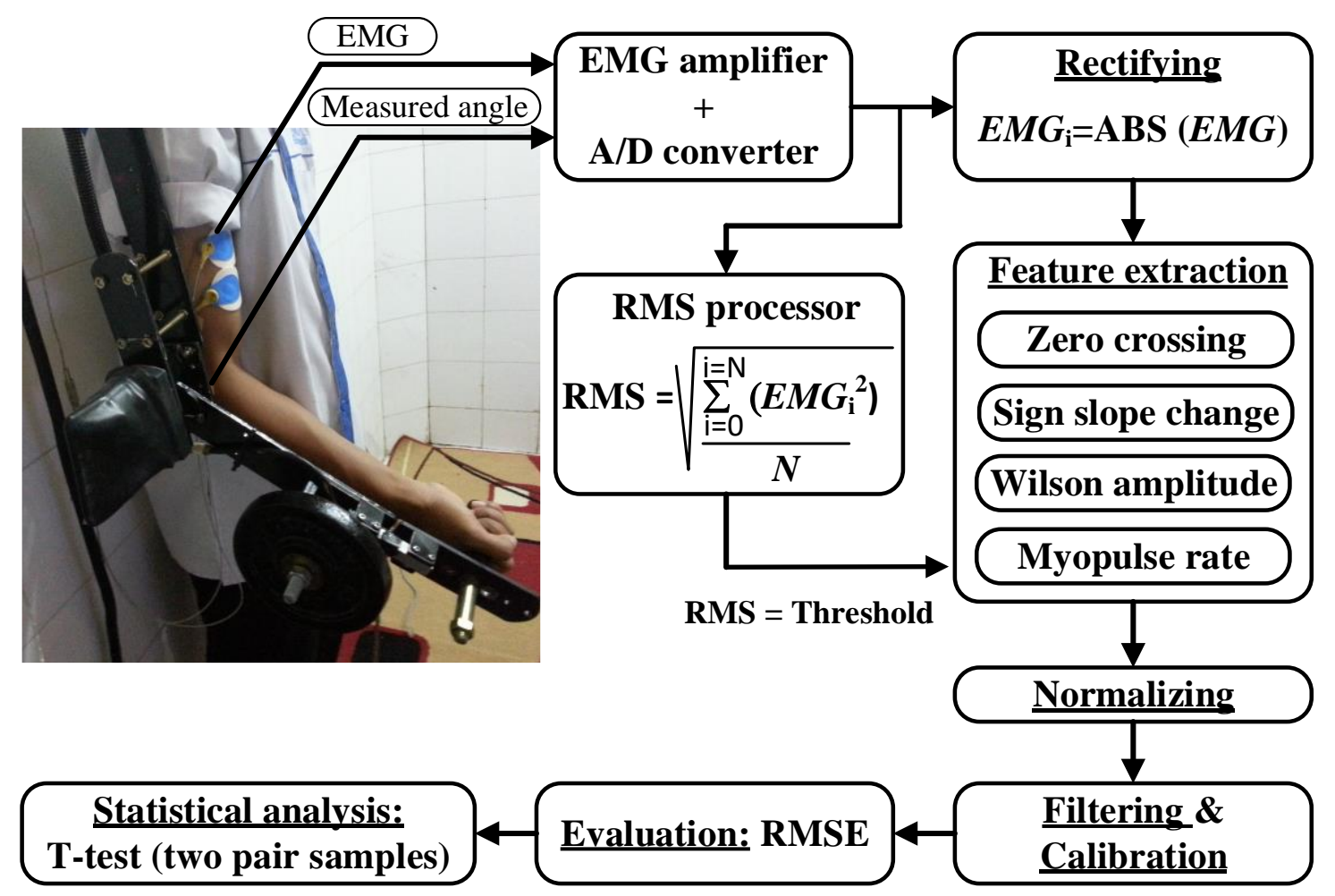

Figure 1. The experiment protocol of the data collection.

The exoskeleton frame is used to synchronize the elbow joint motion (flexion and extension) (Figure 1). In this work, in the initial time of the data collection, the subjects were assumed in the non-fatigue condition. Before the data collection is performed, subjects suggested not to do any heavy activity as stated in the form of consents of experiment protocols. The subjects were asked to move the elbow from extension to flexion motion repetitively within the range of $0^{\circ}$ to $140^{\circ}$, The subjects had to maintain the motion (flexion and extension) from non-fatigue to fatigue condition in fifteen minutes or the subjects can stop the motion if they experienced a discomfort condition in the biceps. In this study, the standard protocol used to assess muscle fatigue followed the procedures which had been performed by previous studies [32, 33]. The EMG signal was amplified using an amplifier with a bandpass filter of 20 to $500 \mathrm{~Hz}$ and notch filter of $50 \mathrm{~Hz}$. The EMG signal was sampled with a sampling frequency of $1000 \mathrm{~Hz}$, this is according to the Nyquist rule [34]. The real angle of the elbow-joint was measured using a linear potentiometer and filtered using a low pass filter. The EMG and elbow-joint angle were recorded using data acquisition system and standard computer (Intel Core i3-3217U CPU at $1.80 \mathrm{GHz}, 8$ GB of RAM, Windows 8).

\section{Data Processing, Evaluation, and Statistical Analysis}

The EMG data processing was consisted of rectifying, time-domain features extraction, features normalization, filtering, calibration, evaluation and statistical analysis (Figure 1). The EMG data along with the measured angle were processed from non-fatigue to fatigue condition up to 15 minutes. The EMG signal was extracted using four standard time-domain features and four 
modified features. The features were selected which have a threshold parameter. The EMG signal was segmented and extracted with a window length (WL) of 100 milliseconds. These features are zero crossing (ZC), sign slope change (SSC), Wilson amplitude (WAMP) and myopulse percentage rate (MYOP) as written in Table 1 [16, 27]. Commonly the threshold-values of these features were set by a real constant value [35-37]. Over a long period of contraction in dynamic motion (flexion and extension), the muscle fatigue would appear and the power of EMG also increased significantly as mention in the previous studies [23, 26, 27]. In order the features able to adapt to the fatigue condition, the threshold values needed to be adjusted adaptively so that the output of the estimation could be maintained as in the non-fatigue condition. In this study, the threshold value was calculated based on the root mean square (RMS) of the EMG signal. This RMS feature is commonly used by previous studies to detect muscle fatigue using EMG signal [38]. In order the threshold parameter could adapt to the EMG signal continuously, the average of RMS value was calculated based on each cycles of motion.

Table 1. Time domain feature extraction based on frequency [27]

\begin{tabular}{|c|c|c|c|}
\hline No & Features & No & Features \\
\hline \multirow{7}{*}{$\begin{array}{l}1 . \\
2 . \\
2 .\end{array}$} & Zero crossing: & 3. & Wilson amplitude \\
\hline & $Z C=\sum_{i=1}^{N-1}\left[f\left(x_{i} \times x_{i+1}\right) \cap\left|x_{i}-x_{i+1}\right| \geq\right.$ threshold & & $W A M P=\sum_{i=1}^{N-1}\left[f\left(\left|x_{i}-x_{i+1}\right|\right)\right]$ \\
\hline & $f(x)=\left\{\begin{array}{l}1, \text { if } \rightarrow x \geq \text { threshold } \\
\text {. }\end{array}\right.$ & & $f(x)=\left\{\begin{array}{l}1, \text { if } \rightarrow x \geq \text { threshold } \\
\text {. }\end{array}\right.$ \\
\hline & 0, otherwise & 4. & 0 ,otherwise \\
\hline & Sign slope change: & & Myopulse percentage rate \\
\hline & $S S C=\sum_{i=1}^{N-1}\left[f\left[\left(x_{i}-x_{i-1}\right) \times\left(x_{i}-x_{i+1}\right)\right]\right]$ & & $M Y O P=\frac{1}{N} \sum_{i=1}^{N}\left[f\left(x_{i}\right)\right]$ \\
\hline & $f(x)=\left\{\begin{array}{c}1, \text { if } \rightarrow x \geq \text { threshold } \\
0, \text { otherwise }\end{array}\right.$ & & $f(x)=\left\{\begin{array}{l}1, \text { if } \rightarrow x \geq \text { threshold } \\
0, \text { otherwise }\end{array}\right.$ \\
\hline
\end{tabular}

where $N$ indicates the number of the sample point, $x_{i}$ indicates the EMG signal, threshold indicates the level of amplitude that limited the signal to be processed. Figure 2 shows the EMG signal, measured angle, calculating the threshold from RMS, and feature extraction process using zero crossing feature with an adaptive threshold.

In the proposed method, the threshold of the features (Figure 2) was calculated using the root mean square (RMS). Since the RMS can represent the power of the EMG signal, it can be used to detect the effect of muscle fatigue on the EMG signal [38, 44-48]. The RMS value was calculated based on each cycle of motion and then the value was used in the next feature extraction process as an adaptive threshold. The RMS values were calculated according to the Eq. (1) as follow:

$$
T h[m]=R M S=\sqrt{\frac{1}{N} \sum_{i=1}^{N} x_{i}^{2}}
$$

where $\mathrm{N}$ is the number of samples (or window length in milliseconds) and $x_{\mathrm{i}}$ is the EMG sample at discrete-time index $i$ (in $\mathrm{mV}$ ), and $T h[m]$ is the adaptive threshold for the $\mathrm{m}^{\text {th }}$ cycles. The threshold parameter of the ZC, SSC, WAMP, and MYOP features (Table 1) can be substituted with Eq. (1). 


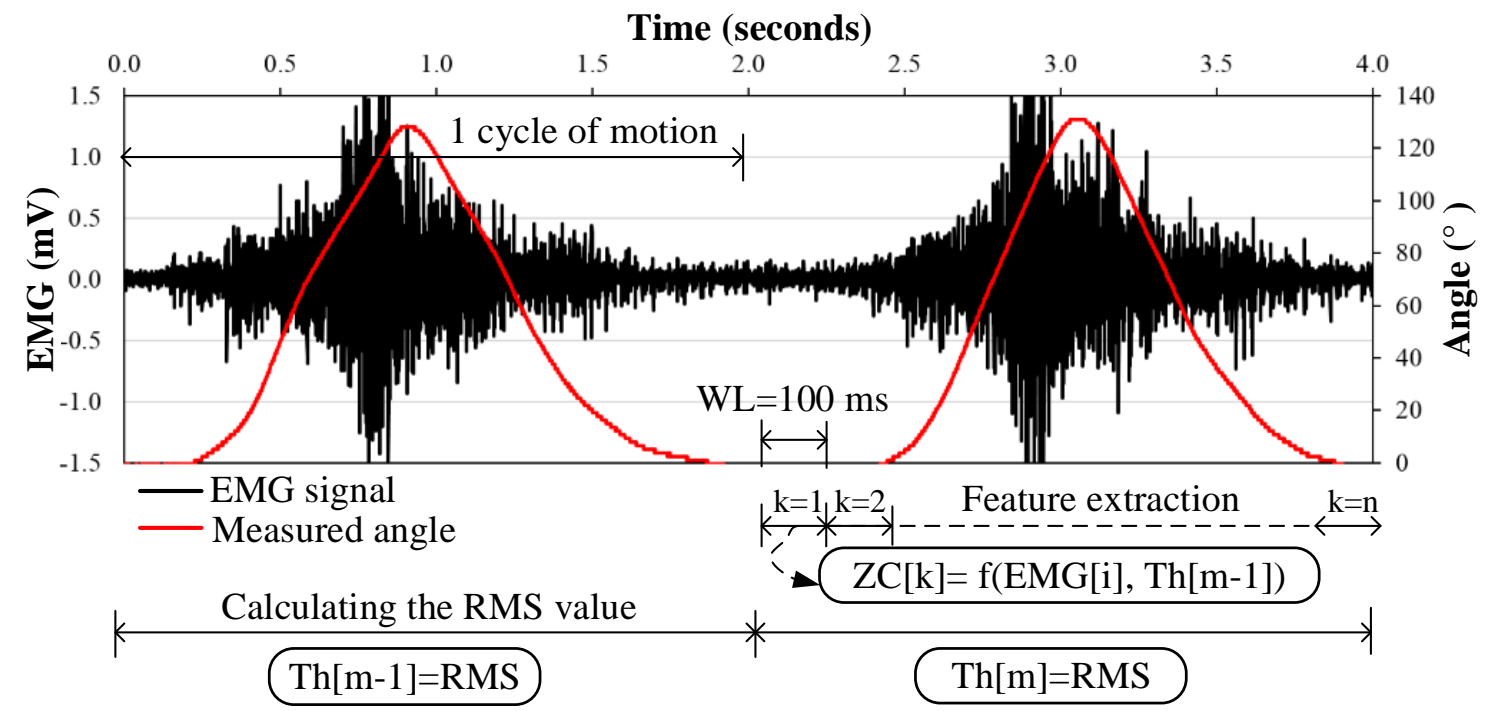

Figure 2. The representation of the ZC feature with an adaptive threshold. The EMG signal is extracted for each window length (WL) or segmented window.

Equation (2) represented a new ZC feature with an adaptive threshold after modifying the threshold parameter.

$$
\begin{aligned}
& Z C[k]=\sum_{i=1}^{N-1}\left[f\left(x_{i} \times x_{i+1}\right) \cap\left|x_{i}-x_{i+1}\right| \geq \operatorname{Th}[m-1]\right. \\
& f(x)=\left\{\begin{array}{c}
1, \text { if } \rightarrow x \geq \operatorname{Th}[m-1] \\
0, \text { otherwise }
\end{array}\right.
\end{aligned}
$$

where $Z C[k]$ is the feature for the- $k$ segment (window), $x_{\mathrm{i}}$ is the EMG sample at discrete-time index $i$, and $T h[m]$ is the adaptive threshold which generated from the RMS of the EMG signal. Since each feature resulted has a different range value, the features need to be normalized. After the normalization process, the low pass filter was applied in order to reduce the noises. In this study, the filtering process used the second order Butterworth low pass filter. The filter was designed using infinite impulse response (IIR) as shown in the Eq. (3) [34].

$$
y[n]=b_{0} x[n]+b_{1} x[n-1]+. .+b_{P} x[n-P]-a_{1} y[n-1]-a_{2} y[n-2]-. .-a_{Q} y[n-P]
$$

where $x[n]$ denotes the $n^{\text {th }}$ input sample, $y[n]$ denotes $n^{\text {th }}$ output sample, $b_{0}, b_{1}, b_{M}, a_{1}, a_{2}, a_{N}$ are the filter coefficients, and $P=Q$ is the filter order. Previous studies used this filtering technique to smooth the estimation [17, 42]. In this study, the coefficients of low pass filter, the feature extraction, and low pass filtering was performed using MATLAB (Student version, Math Works, Inc., USA). The low pass filter was designed with a cutoff frequency of $80 \mathrm{~Hz}$. The calibration was performed to return the unit of the estimation in degree unit. The calibration process is conducted by multiplying the estimated angle (the output of the filtering process) with the maximum value of the range of motion (in this case is $140^{\circ}$ ). The performance of the proposed and standar method was evaluated using the root mean square error (RMSE) as a standard measurement of the elbow joint angle estimation $[10,12,13]$. The detail of the comparison between the proposed and standard method is shown in Figure 3. 


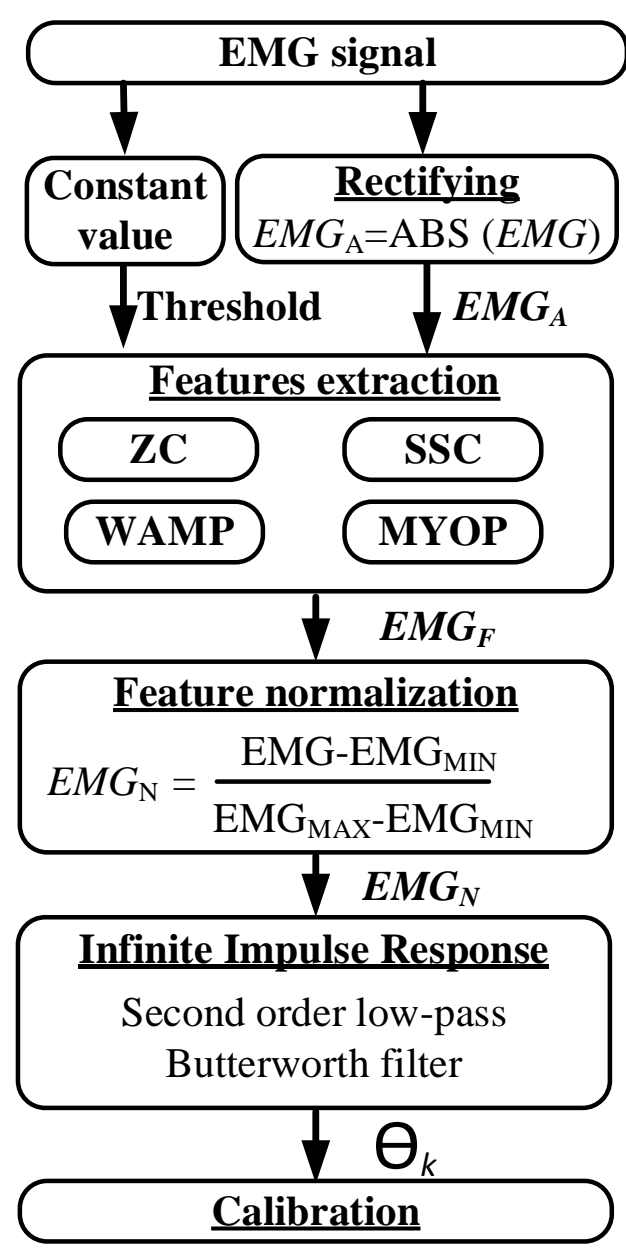

(a) Standard method

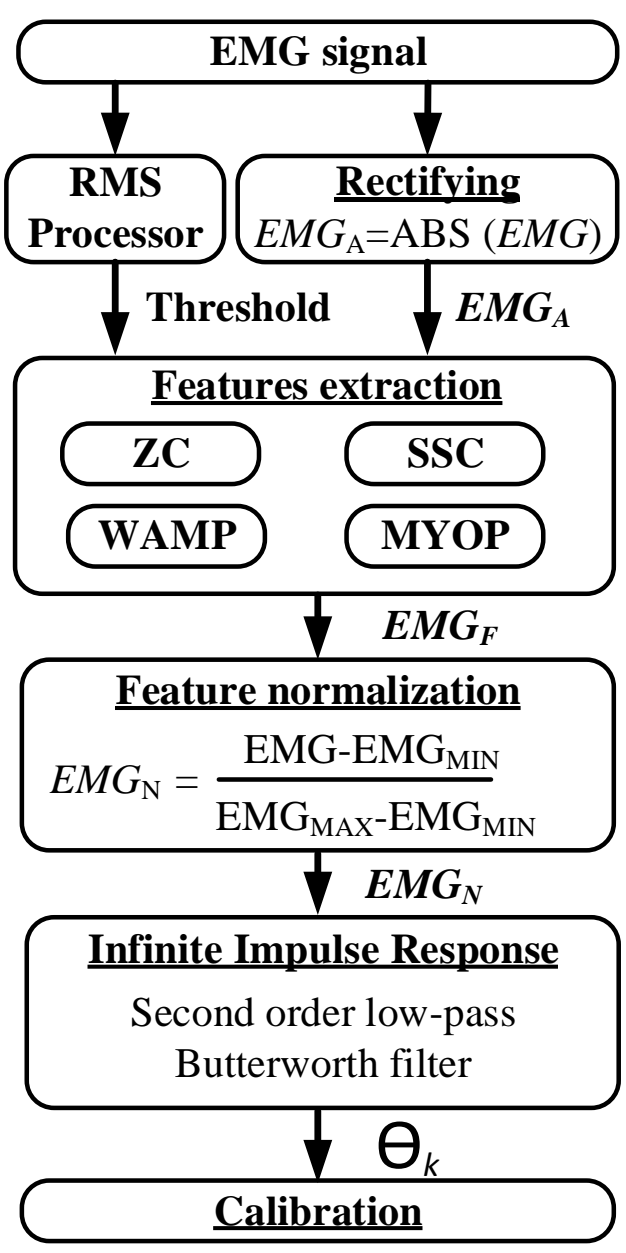

(b) Proposed method

Figure 3. The comparison of method to estimate the elbow-joint angle between (a) standard method and (b) proposed method.

In the standard method as shown in Figure 3(a), the threshold was defined in the first section before the pre-processing with a certain real value. In the proposed method as shown in Figure 3(b), the threshold was calculated before in the pre-processing section. The adaptive threshold was calculated based on the RMS value in each cycle of motion in the elbow flexion and extension so that the feature able to adapt in the non-fatigue and fatigue condition.

The performance of the proposed and standard methods was analyzed using two pair samples T-test with the alpha value is 0.05 . The $\mathrm{p}$-value can indicate whether the RMSE values are significant ( $p$-value $<0.05$ ) or insignificant difference ( $p$-value $>0.05$ )

\section{RESULTS AND DISCUSSION}

The adaptive threshold that we developed in the proposed method could compensate the effect of the muscles fatigue. Based on evaluation of the performance of the elbow joint angle estimation, we found that the proposed method has better performance than the standard method. Statistical analysis showed that there was a significant improvement of the RMSE between the proposed and standard method ( $\mathrm{p}$-value $<0.05$ ). The representation of the elbow joint angle estimation (from feature SSC) resulted from the standard and proposed method is shown in Figure 4. 

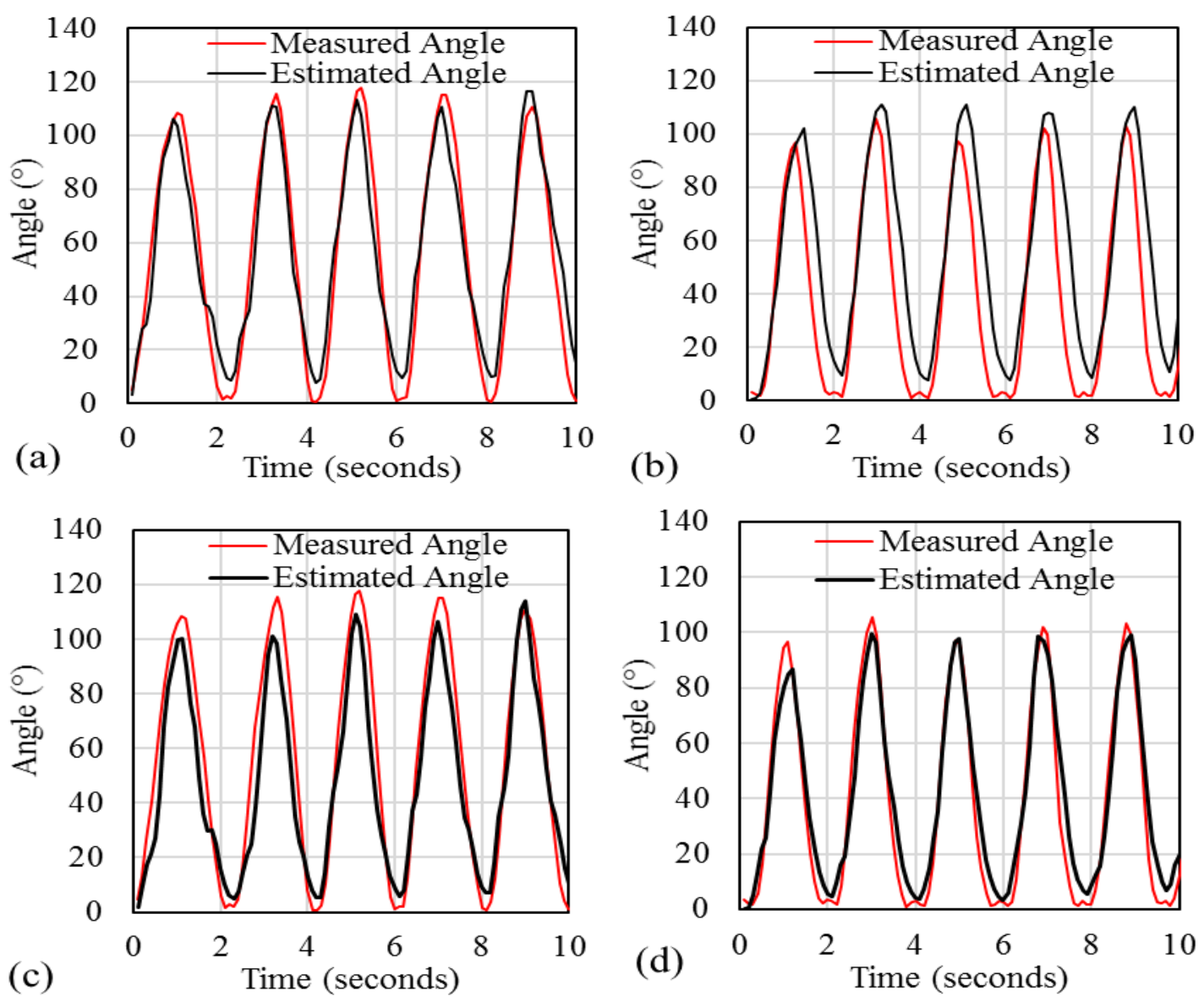

Figure 4. The representation of the estimated angles from modified SSC feature. The estimated angles which resulted from the standard method for (a) non-fatigue and (b) fatigue condition. The estimated angles which resulted from the proposed method for (c) non-fatigue and (d) fatigue condition (from subject C).

In the standard and proposed method (Figure 4), the estimated angles are able to follow the measured angle but with various performance. The RMSE values of the estimated angle as shown in Figure 4 (a), (b), (c), and (d) are $17.61^{\circ}, 26.28^{\circ}, 13.96^{\circ}$, and $16.27^{\circ}$, respectively. When the muscle is in the fatigue condition, the estimated angle tends to shift from the measured angle and its values are relatively higher than the measured angle as shown in Figure 4 (b). However, the proposed method was able to compensate the effect of muscle fatigue. It was shown that the estimated and measured angle was almost coincided [Figure 4(d)]. Furthermore, the RMSE values obtained from non-fatigue to fatigue condition from all subjects were pooled to perform a descriptive statistic. The RMSE values were grouped based on the standard and proposed method for each feature. The statistic descriptive was conducted in order to obtain the mean and standard deviation of the RMSE for all subjects. Figure 5 shows the boxplot diagram of the RMSE for comparison between standard and proposed method (all features). In the standard method [Figure 5(a)], the variability of the RMSE values is wider for all features if it is compared to the proposed method [Figure 5(b)]. This indicated that the proposed method was able to reduce the variability of the RMSE values [Figure 5(b)]. The comparison of the boxplot among the features was found that features SSC has better performance if it is compared to the other features both for the standard and proposed method. 
(a) Standard method

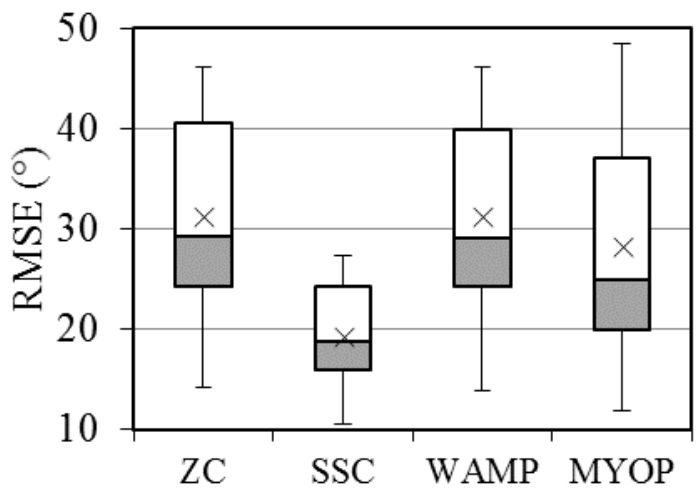

(b) Proposed method

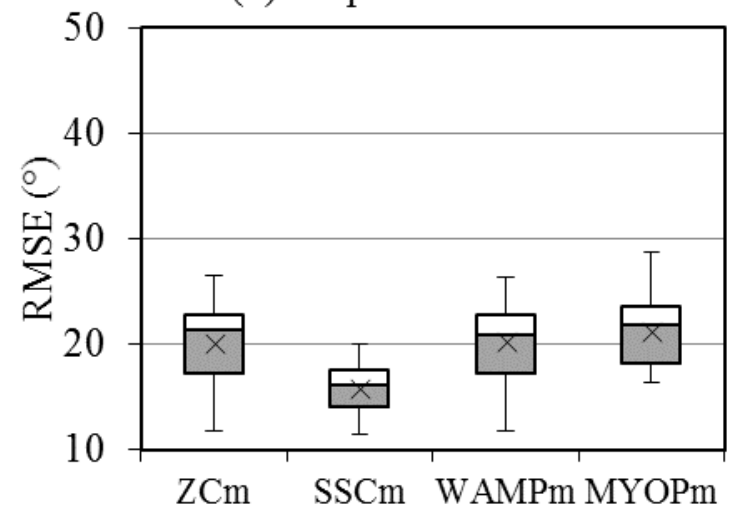

Figure 5. The comparison of RMSE between (a) the standard method and (b) proposed method for all features. The RMSE values is evaluated from non-fatigue to fatigue condition. The $\mathrm{m}$-index indicates the modified feature of ZC, SSC, WAMP, and MYOP (for all subjects). The cross sign in the box indicates the mean values.

The summary of statistics descriptive (mean and standard deviation) of the RMSE values is shown in Table 2. The significant difference of RMSE between proposed and standard method was examined using two paired sample T-test. The alpha value was chosen as 0.05 (95\% confidence). The p-value indicated whether there is a significant difference of RMSE or not. When the p-value is less than 0.05, it indicated that there is a significant difference of RMSE and on the contrary. Table 2 shows that there is a significant difference of RMSE between the standard and proposed method for all features (ZC, SSC, WAMP, and MYOP) which the p-values are <0.001). Furthermore, Table 2 shows that the proposed method (all features) can maintain the performance with a small standard deviation which ranged between $2.39^{\circ}$ and $3.76^{\circ}$. In the standard method, the standard deviation ranged between $5.19^{\circ}$ and $11.39^{\circ}$ for all features. In this study, it is found that the SSC feature has the best performance to estimate the elbow joint angle using EMG signal with muscle fatigue compensation and the estimation is conducted only using single muscle group from biceps muscle.

Table 2. The summary of RMSE (mean \pm standard deviation) and p-value for standard and proposed method $(\alpha=0.05)$. The RMSE value is calculated from non-fatigue to fatigue condition for all subjects.

\begin{tabular}{lccc}
\hline \multicolumn{1}{c}{ Features } & Standard method & Proposed method & p-value \\
\hline ZC & $32.36^{\circ} \pm 9.60^{\circ}$ & $20.26^{\circ} \pm 3.76^{\circ}$ & $<0.001$ \\
SSC & $19.29^{\circ} \pm 5.19^{\circ}$ & $15.93^{\circ} \pm 2.39^{\circ}$ & $<0.001$ \\
WAMP & $31.39^{\circ} \pm 9.70^{\circ}$ & $20.28^{\circ} \pm 3.73^{\circ}$ & $<0.001$ \\
MYOP & $28.34^{\circ} \pm 11.39^{\circ}$ & $21.35^{\circ} \pm 3.64^{\circ}$ & $<0.001$ \\
\hline
\end{tabular}

In order to justify the proposed method, the results of this study are compared to the other related studies. Tang proposed an elbow-joint angle estimation based on backpropagation artificial neural network method using EMG signal [10]. He obtained that the RMSE values ranged between $9.67^{\circ}$ and $12.42^{\circ}$. However, the experiment is only conducted on one subject and the EMG signal is collected from four muscles, namely: biceps, triceps, brachioradialis, and anconeus. Pau investigated the elbow-joint angle estimation using Hill-based muscle model and optimized the estimate results using a Genetic algorithm [12]. The EMG was extracted from two muscles (biceps and triceps). He found that the RMSE (mean \pm SD.) for multi-cycle of motion was $22.00^{\circ} \pm 6.6^{\circ}$. However, all of those previous methods did not consider to compensate the effect of the muscle fatigue. Thilina developed a neuro-fuzzy modifier to compensate the effect of muscle fatigue using 
mean power frequency [25]. He found that the error of estimated elbow joint angle was between $5^{\circ}$ and $15^{\circ}$. Na proposed an elbow joint force with muscle fatigue compensation using EMG signal based on twitch muscle model. The \%RMSE (mean \pm SD.) for the dynamic motion was $15,6 \pm 24,7$ [24]. Although significant results are found in this study, however, this work is only dealing with an elbow flexion and extension motion. A new method should be proposed if it is used to estimate other motion (such as pronation and supination) and more degree of freedom so that the proposed method can be applied in real life. Considering that EMG signal is influenced by muscle fatigue, KMeans clustering [43] approach can be used to classify fatigue levels.

\section{CONCLUSIONS}

In this study, elbow joint angle estimation under non-fatigue and fatigue condition using EMG signal is evaluated with an adaptive threshold in order to compensate the effect of muscle fatigue. The adaptive threshold is obtained by extracting the EMG signal using RMS feature. The major contribution of this work is that the proposed method is performed with high performance and consistent results in the non-fatigue and fatigue condition. The performance comparison between standard and proposed method shows that there is a significant difference of RMSE (p-value $<0.05$ ). Based on performance evaluation of all features, the modified SSC feature result in better performance than the others. The major difference to estimated the elbow joint angle in this work compared to the others is that the proposed method only uses single muscle (bicep). In the future work, the proposed method can be used in the control algorithm for development of exoskeleton and prosthetic devices so that the system able to estimate the elbow joint angle accurately and to compensate the effect of muscle fatigue using EMG signal.

\section{ACKNOWLEDGEMENTS}

The authors would like to thank to the Gadjah Mada University and Polytechnic of Health Surabaya for laboratory facilities and financial assistance under education grant from Ministry of Health, Republic of Indonesia No. HK.02.02/H. V/SK/360/2015.

\section{REFERENCES}

[1] Nishihara K, Isho T. Location of electrodes in surface EMG. EMG Methods for Evaluating Muscle and Nerve Function, InTech. 2012; 17-30.

[2] Merlo A, Campanini I. Technical aspects of surface electromyography for clinicians. The Open Rehabilitation Journal. 2010; 3: 98-109.

[3] Oskoei MA, Hu H. Myoelectric control systems - a survey. Biomedical Signal Processing and Control. 2007; 2 (4): 275-294.

[4] Antuvan CW, Ison M., and Artemiadis P. Embedded human control of robots using myoelectric interfaces. IEEE Transactions on Neural Systems and Rehabilitation Engineering. 2014; 22 (4): 820-827.

[5] Fougner A, Stavdahl O, Kyberd PJ, Losier YG, Parker PA. Control of upper limb prostheses: terminology and proportional myoelectric control - A review. IEEE Transactions on Neural Systems and Rehabilitation Engineering. 2012; 20 (5): 663-677.

[6] Zhang X, Liu Y, Zhang F, Ren J, Lindsay Y, Yang Q, Huang H. On design and implementation of neural-machine interface for artificial legs. IEEE Transactions on Industrial Informatics. 2012; 8 (2): 418-429.

[7] Chin LC, Nisa bin Basah S, Yaacob S, Juan YE. Conceptual design and implementation for visual tracking ankle rehabilitation system. Journal of Mechanical Engineering and Sciences. 2014; 7: 1208-1218. 
[8] Fernini B, Temmar M, Noor M. Toward a dynamic analysis of bipedal robots inspired by human leg muscles. Journal of Mechanical Engineering and Sciences. 2018; 12 (2): 35933604.

[9] Rath AK, Das HC, Parhi DR, Kumar PB. Application of artificial neural network for control and navigation of humanoid robot. Journal of Mechanical Engineering and Sciences. 2018; 12 (2): 3529-3538.

[10] Tang Z, Zhang K, Sun S, Gao Z, Zhang L, Yang Z. An upper-limb power-assist exoskeleton using proportional myoelectric control. Sensors. 2014; 14 (4): 6677-6694.

[11] Li Q, Song Y, Hou Z, Zhu B. sEMG based joint angle estimation of lower limbs using LSSVM. Lecture Notes in Computer Science (including subseries Lecture Notes in Artificial Intelligence and Lecture Notes in Bioinformatics). 2013; 8226 (1): 292-300.

[12] Pau JWL, Xie SSQ, Pullan AJ. Neuromuscular interfacing: establishing an EMG-driven model for the human elbow joint. IEEE Transactions on Biomedical Engineering. 2012; 59 (9): 2586-2593.

[13] Pang M, Guo S, Huang Q, Ishihara H, Hirata H. Electromyography-based quantitative representation method for upper-limb elbow joint angle in sagittal plane. Journal of Medical and Biological Engineering. 2015; 35 (2): 165-177.

[14] Kiguchi K, Hayashi Y. EMG-based control of a lower-limb power-assist robot. Intelligent Assistive Robots. Springer Tracts in Advanced Robotics 106. Springer. 2015; 371-383.

[15] Li Z, Wang B, Sun F, Yang C, Xie Q, Zhang W. SEMG-based joint force control for an upper-limb power-assist exoskeleton robot. IEEE Journal of Biomedical and Health Informatics. 2014; 18 (3): 1043-1050.

[16] Triwiyanto T, Wahyunggoro O, Nugroho H. A, Herianto. An investigation into time domain features of surface electromyography to estimate the elbow joint angle. Advances in Electrical and Electronic Engineering. 2017; 15 (3): 448-458.

[17] Jang G, Kim J, Choi Y, Yim J. Human shoulder motion extraction using EMG signals. International Journal of Precision Engineering and Manufacturing. 2014; 15 (10): 21852192.

[18] Triwiyanto T, Wahyunggoro O, Nugroho HA, Herianto H. Evaluating the performance of Kalman filter on elbow joint angle prediction based on electromyography. International Journal of Precision Engineering and Manufacturing. 2017; 18 (12): 1739-1748.

[19] Al-Mulla MR, Sepulveda F, Colley M. sEMG techniques to detect and predict localised muscle fatigue. EMG Methods for Evaluating Muscle and Nerve Function. 2012:1-532.

[20] Basmajian JV, de Luca CJ. Chapter 8. Muscle Fatigue and Time-Dependent Parameters of the Surface EMG Signal. Muscles alive: their functions revealed by electromyography. Baltimore: Williams \& Wilkins. 1985; 201-222.

[21] Gonzalez-Izal M, Malanda A, Gorostiaga E, Izquierdo M. Electromyographic models to assess muscle fatigue. Journal of Electromyography and Kinesiology. 2012; 22 (4): 501-512.

[22] Chowdhury SK, Nimbarte AD, Jaridi M, Creese RC. Discrete wavelet transform analysis of surface electromyography for the fatigue assessment of neck and shoulder muscles. Journal of Electromyography and Kinesiology. 2013; 23 (5): 995-1003.

[23] Triwiyanto T, Wahyunggoro O, Nugroho HA, Herianto H. Continuous wavelet transform analysis of surface electromyography for muscle fatigue assessment on the elbow joint motion. Advances in Electrical and Electronic Engineering. 2017; 15 (3): 424-434.

[24] Na Y, Kim J. Dynamic elbow flexion force estimation through a muscle twitch model and sEMG in a fatigue condition. IEEE Transactions on Neural Systems and Rehabilitation Engineering. 2017; 25 (9): 1431-1439.

[25] Lalitharatne TD, Teramoto K, Hayashi Y, Nanayakkara T, Kiguchi K. Evaluation of fuzzyneuro modifiers for compensation of the effects of muscle fatigue on EMG-based control to be used in upper-limb power-assist exoskeletons. Journal of Advanced Mechanical Design, 
Systems, and Manufacturing. 2013; 7 (4): 736-751.

[26] Yahya AB, Mohd Bukhari W, Horng CS, Sudirman R. Electromyography signal on biceps muscle in time domain analysis. Journal of Mechanical Engineering and Sciences. 2014; 7 : 1179-1188.

[27] Hakonen M, Piitulainen H, Visala A. Biomedical signal processing and control current state of digital signal processing in myoelectric interfaces and related applications. Biomedical Signal Processing and Control. 2015; (18): 334-359.

[28] Triwiyanto T, Wahyunggoro O, Nugroho HA, Herianto H. DWT analysis of sEMG for muscle fatigue assessment of dynamic motion flexion-extension of elbow joint. 8th International Conference on Information Technology and Electrical Engineering (ICITEE), 2016; $1-6$.

[29] Martini FH, Ober WC. Fundamentals of Anatomy and Physiology. Englewood Cliffs, New Jersey 7632: Prentice Hall. 1995.

[30] Lenzi T, De Rossi SMM, Vitiello N, Carrozza MC. Intention-based EMG control for powered exoskeletons. IEEE Transactions on Biomedical Engineering. 2012; 59 (8): 21802190.

[31] Stegeman D, Hermens H. Standards for surface electromyography: the european project surface EMG for non-invasive assessment of muscles (SENIAM). 2007; 108-112.

[32] Potvin JR. Effects of muscle kinematics on surface EMG amplitude and frequency during fatiguing dynamic contractions. Journal of Applied Physiology. 1997; 82(1): 144-151.

[33] Potvin JR, Bent LR. A validation of techniques using surface EMG signals from dynamic contractions to quantify muscle fatigue during repetitive tasks. Journal of Electromyography and Kinesiology. 1997; 7 (2): 131-139.

[34] Tan L, Jiang J. Digital signal processing: fundamental and applications. Academic Press. 2013.

[35] Hudgins B, Parker P, Scott RN. A new strategy for multifunction myoelectric control. IEEE Transactions on Biomedical Engineering. 1993; 40 (1): 82-94.

[36] Du Y, Lin C, Shyu L, Chen T. Portable hand motion classifier for multi-channel surface electromyography recognition using grey relational analysis. Expert Systems with Applications. 2010; 37 (6): 4283-4291.

[37] Zardoshti-Kermani M, Wheeler BC, Badie K, Hashemi RM. EMG feature evaluation for movement control of upper extremity prostheses. IEEE Transactions on Rehabilitation Engineering. 1995; 3 (4): 324-333.

[38] Fukuda TY, Echeimberg JO, Pompeu JE, Lucareli PRG, Garbelotti S, Gimenes RO, Apolinario A. Root mean square value of the electromyographic signal in the isometric torque of the quadriceps, hamstrings and brachial biceps muscles in female subjects. Journal of Applied Research. 2010; 10 (1): 32-39.

[39] Basmajian J, De Luca CJ. Description and analysis of the EMG signal. Muscles alive: their functions revealed by electromyography. 1985; 65-100.

[40] Al-Mulla MR, Sepulveda F, Al-Bader B. Optimal elbow angle for extracting sEMG signals during fatiguing dynamic contraction. Computers. 2015; 4: 251-264.

[41] Broman H, Bilotto G, De Luca CJ. Myoelectric signal conduction velocity and spectral parameters: influence of force and time. Journal of applied physiology. 1985; 58 (5): 14281437.

[42] Yu HJ, Lee AY, Choi Y. Human elbow joint angle estimation using electromyogram signal processing. IET Signal Process. 2011; 5 (8): 767-775.

[43] Yunoh MFM, Abdullah S, Saad MHM, Nopiah ZM, Nuawi MZ. Fatigue feature extraction analysis based on a K-means clustering approach. Journal of Mechanical Engineering and Sciences. 2015; 8: 1275-1282. 